Review

\title{
The CTM4XAS program for EELS and XAS spectral shape analysis of transition metal L edges
}

\author{
Eli Stavitski ${ }^{1}$, Frank M.F. de Groot* \\ Inorganic Chemistry and Catalysis, Utrecht University, Sorbonnelaan 16, 3584 CA Utrecht, Netherlands
}

\section{A R T I C L E I N F O}

\section{Article history:}

Received 21 December 2009

Received in revised form 18 June 2010

Accepted 18 June 2010

\section{PACS:}

78.70Dm

\section{Keywords:}

X-ray Absorption Spectroscopy

EELS

ELNES

XANES

L edges

Transition metal compounds

\begin{abstract}
A B S T R A C T
The CTM4XAS program for the analysis of transition metal L edge Electron Energy Loss Spectroscopy (EELS) or X-ray Absorption Spectra (XAS) is explained. The physical background of the calculations is briefly discussed. The program consists of three theoretical components, based on, respectively, atomic multiplet theory, crystal field theory and charge transfer theory. The theoretical concepts are explained and a number of examples are presented. The calculation of the 2p EELS and XAS spectra of transition metal ions, is given in detail, including their Magnetic Circular Dichroism (MCD). In addition, examples of 1 s, 2s, 3s, 2p and 3p X-ray Photoemission Spectroscopy (XPS) are given.
\end{abstract}

(C) 2010 Elsevier Ltd. All rights reserved.

\section{Contents}

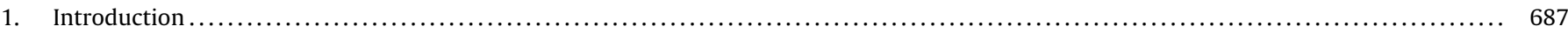

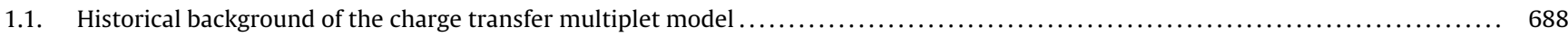

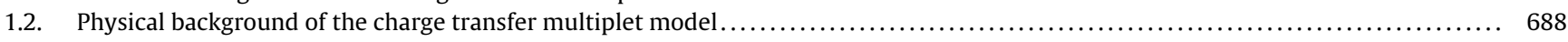

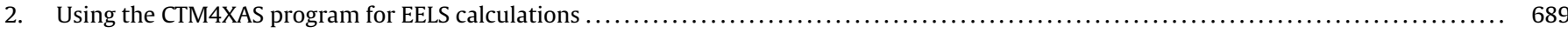

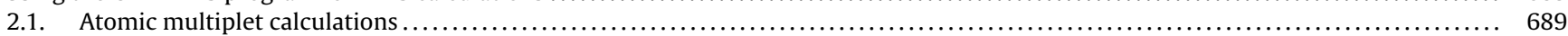

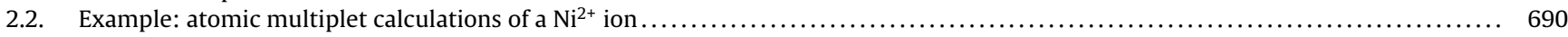

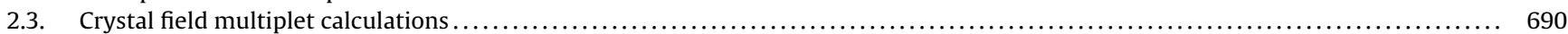

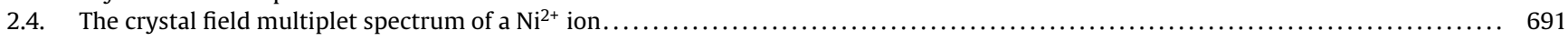

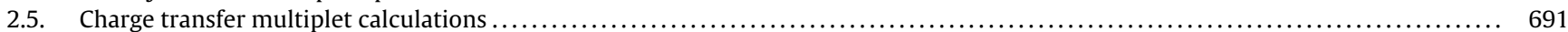

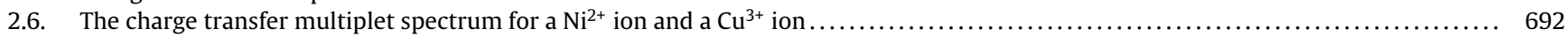

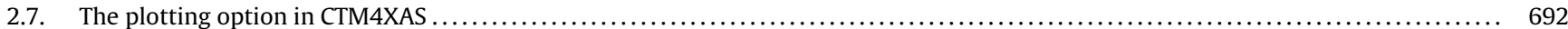

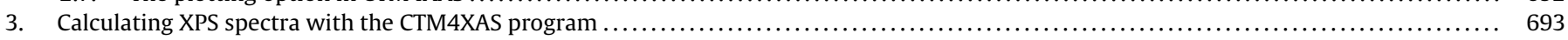

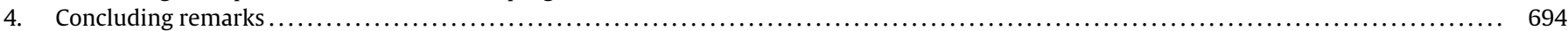

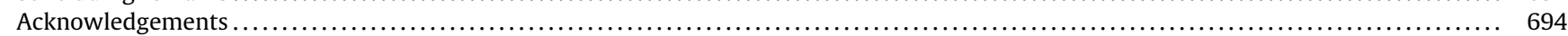

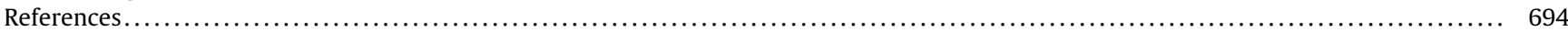

\section{Introduction}

This paper discusses the charge transfer multiplet program for the analysis of transition metal $2 \mathrm{p}$ and $3 \mathrm{p}$ core level excitations,

\footnotetext{
* Corresponding author. Tel.: +31 302536763; fax: +31 302511027.

E-mail address: f.m.f.degroot@uu.nl (F.M.F. de Groot).

1 Current address: National Synchrotron Light Source, Brookhaven National Laboratory, Upton, NY 11973, USA.
}

either Electron Energy Loss Spectroscopy (EELS) or X-ray Absorption Spectroscopy (XAS). In addition, a detailed introduction to the CTM4XAS analysis software of transition metal core level spectra is given. CTM4XAS stands for Charge Transfer Multiplet program for $\mathrm{X}$-ray Absorption Spectroscopy. An important aspect that determines the amount of detail in an EELS or XAS spectrum is the lifetime broadening of the core hole, which is typically $0.2 \mathrm{eV}$ half-width half-maximum for $\mathrm{L}_{3}$ edges. The experimental resolution must ideally be equal or better than this lifetime broadening. Concerning XAS spectra, an important step was made in eighties 
when the resolution of $0.2 \mathrm{eV}$ was achieved with the development of the SX700 and DRAGON monochromators (Chen and Sette, 1989). In case of EELS spectrometers, the present day resolution of EELS measurements varies between $30 \mathrm{meV}$ in high-resolution EELS (Krivanek et al., 2009) to $1.0 \mathrm{eV}$ or lower at non-dedicated microscopes. We assume that the spectral shape of XAS and EELS is identical and in the remainder of this paper limit ourselves to the discussion of EELS spectra.

\subsection{Historical background of the charge transfer multiplet model}

The two important components of the charge transfer multiplet model are (1) multiplet effects and (2) charge transfer effects. The recognition that the X-ray Absorption Spectra of transition metal and rare earth systems were dominated by atomic effects was first made in the sixties for the $\mathrm{M}_{4,5}$ and $\mathrm{N}_{4,5}$ edges of rare earths (Williams, 1966; Fomichev et al., 1967). The first high-resolution spectra of transition metal systems were performed by Nakai et al. (1974). They assigned part of the spectrum as multiplet structure. Shin et al. (1981) re-assigned the spectra to $3 \mathrm{~d}^{\mathrm{N}} \rightarrow 3 \mathrm{p}^{5} 3 \mathrm{~d}^{N+1}$ multiplet structures. The origin of the multiplet theory for L edges can be traced back to initial developments in Canada (Gupta and Sen, 1974, 1975) and Japan (Suga et al., 1982; Shin et al., 1982). Kotani and Toyozawa (1974) developed the charge transfer effects for mixed valence rare earth systems. Already Asada and Sugano (1976) combined multiplet effects with charge transfer effects. Charge transfer effects and multiplet effects were also combined by Zaanen et al. (1985).

Within this background, Theo Thole started developing his multiplet code. In 1985 he calculated all rare earth $M_{4,5}$ edge with atomic multiplets. He added the crystal field effects via the group theory program of Butler (1981) and calculated the Ledges of transition metal systems in 1988, with van der Laan and Butler (Thole et al., 1988). Systematic studies of the transition metal Ledges were performed with de Groot et al. (de Groot et al., 1990a,b) and by van der Laan (van der Laan, 1991; van der Laan and Kirkman, 1992). The combination of Theo Thole's crystal field multiplet calculations with charge transfer effects and Auger matrix elements was programmed by Theo Thole and Ogasawara in 1991, initially for mixed valence rare earth ions (Ogasawara et al., 1991a,b).

We use the charge transfer multiplet model as developed by Theo Thole and with contributions by Ogasawara as the basis for the CTM4XAS calculations. The actual source codes are the charge transfer multiplet calculations where some options have been removed by Ogasawara, where it is noted that the physics of this program has not been changed since 1991 . However, over the last 20 years, many improvements and additions have been added to the charge transfer multiplet program. Some recent developments are mentioned in the next section, in particular with respect to ab initio multiplet calculations.

In conclusion, one should use the CTM4XAS calculations as an initial tool to simulate the L edges of transition metal systems. The symmetry-options are restricted to octahedral and tetragonal and the magnetic field direction is limited to the $z$-axis. More complex phenomena such as additional charge transfer channels in highvalent systems (Hu et al., 1998a,b) or in systems with $\pi$-bonding (Hocking et al., 2006, 2007) are not included. All possibilities for magnetic order are not included and as well as the experiments such as angular and spin-resolved photoemission. We hope that in the future more options can be included and, as a first new tool we are developing a CTM4RIXS software that calculates the two-dimensional Resonant Inelastic X-ray Scattering (RIXS) planes within the charge transfer multiplet model as described in this paper. If one would like to perform more complex calculations this is possible with the range of charge transfer multiplet models that are available or are being developed.

\subsection{Physical background of the charge transfer multiplet model}

The EELS (and XAS) spectral shape is given by the Fermi golden rule. The core electron is excited to an empty state, where at the edge the lowest empty state (allowed by the selection rules) is reached. As such, one essentially probes the empty density of states in the presence of the core hole. Calculations to obtain a quantitative picture of the empty states can be performed with DFT based codes. This includes band structure codes such as PARATEC (Cabaret et al., 2007; Gaudry et al., 2005), PWSCF (Cabaret et al., 2010; Juhin et al., 2010), CASTEP (Gao et al., 2008) or WIEN2K (Schwarz et al., 2002), real space multiple scattering codes such as FDMNES (Joly, 2003) or FEFF (Rehr and Albers, 2000) and 'molecular' DFT codes such as STOBE (Kolczewski and Hermann, 2005) or ORCA (George et al., 2008). The ground state effects of orbital polarization, 3d spin-orbit coupling, the self-interaction correction and the $3 \mathrm{~d} 3 \mathrm{~d}$ correlation energy $U$ are, in general, only partly included in these codes. The creation of a core hole in an EELS experiment creates additional core hole induced effects to the electronic structure. These effects include:

(a) The core hole potential

(b) The core hole spin-orbit coupling

(c) The core hole induced charge transfer effects

(d) The core hole-valence hole exchange interaction.

(e) The core hole-valence hole multipole interactions.

The core hole effects (a) and (b) can be included in DFT calculations, respectively, by introducing an atom with a core hole within a supercell calculations and by performing relativistic DFT calculations. The effects (c), (d) and (e) are usually not (or not completely) included in DFT based calculations. Because of these core hole induced effects, transition metal $\mathrm{L}_{2,3}$ edges can not be calculated with one-electron codes. To date no general approach is available that includes charge transfer and multiplet effects within ab initio codes, either DFT or wave function based. Closest to a generalized ab initio approach are the routes that start with a ground state DFT calculation, project the wave functions (underlying to the charge density) to a small cluster and subsequently solve the charge transfer and multiplet final state effects for such cluster. The methods that are being developed by, for example, Kruger (Kruger and Natoli, 2004), Uozumi (Agui et al., 2009), Ikeno (Ikeno et al., 2009) and Haverkort (Haverkort, 2009) roughly follow such procedure.

In contrast to these ab initio routes, the CTM4XAS program is based on a semi-empirical approach that includes explicitly the important interactions for the calculation of L edge spectra. This includes all the effects discussed above, including the core and valence spin-orbit couplings, the core-valence two-electron integrals (multiplet effects) and the core hole induced charge transfer effects. Calculations based on the charge transfer multiplet programs have been performed over the last 20 years and the main physics behind this approach has been described in a number of reviews and books (de Groot, 1994, 2001,2005; de Groot and Kotani, 2008).

We have written a new program interface that takes care of the performance of charge transfer multiplet calculations. This CTM4XAS program can be downloaded for free (website, 2010). The CTM4XAS program is limited to transition metal spectra and, in addition to XAS/EELS, the program is also capable of calculating the $1 s, 2 s, 3 s, 2 p$ and $3 p$ XPS spectra, $1 s 2 p$ and $1 s 3 p$ XES. An exchange field can be included along the $z$-direction, yielding the corresponding X-MCD spectra for all XAS and XPS spectra calculated. The CTM programs themselves can also be used for other XAS and XPS spectra, and also for X-ray emission (XES) and Auger electron spectroscopy. This includes the experiments performed at resonance: Resonant XES (or Resonant Inelas- 
tic X-ray Scattering, RIXS), resonant photoemission and Auger spectroscopy.

\section{Using the CTM4XAS program for EELS calculations}

Fig. 1 shows the interface window that opens upon starting the CTM4XAS program. From this screen the complete calculation can be performed. The calculated spectrum can be directly shown on screen and is also automatically saved with the name provided. By starting the CTM4XAS program, a number of screens appear. The CTM4XAS screen shows the credits and authors. The main screen that is opened is shown in Fig. 1.

\subsection{Atomic multiplet calculations}

The top part of the screen, shown enlarged in Fig. 2, is used to generate the atomic parameters and/or to perform atomic multiplet calculations. On the top line one has to provide the element that has to be calculated, including its formal charge. This defines the number of occupied d electrons in the ground state. The CTM4XAS program is written for all $3 d, 4 d$ and $5 d$ transition metal ions, including the $3 \mathrm{~d} 0$ systems such as $\mathrm{K}^{+}$and $\mathrm{Ca}^{2+}$. Next to the configuration, one has to choose the experiment one would like to calculate. Options include 1s XAS, 2p XAS

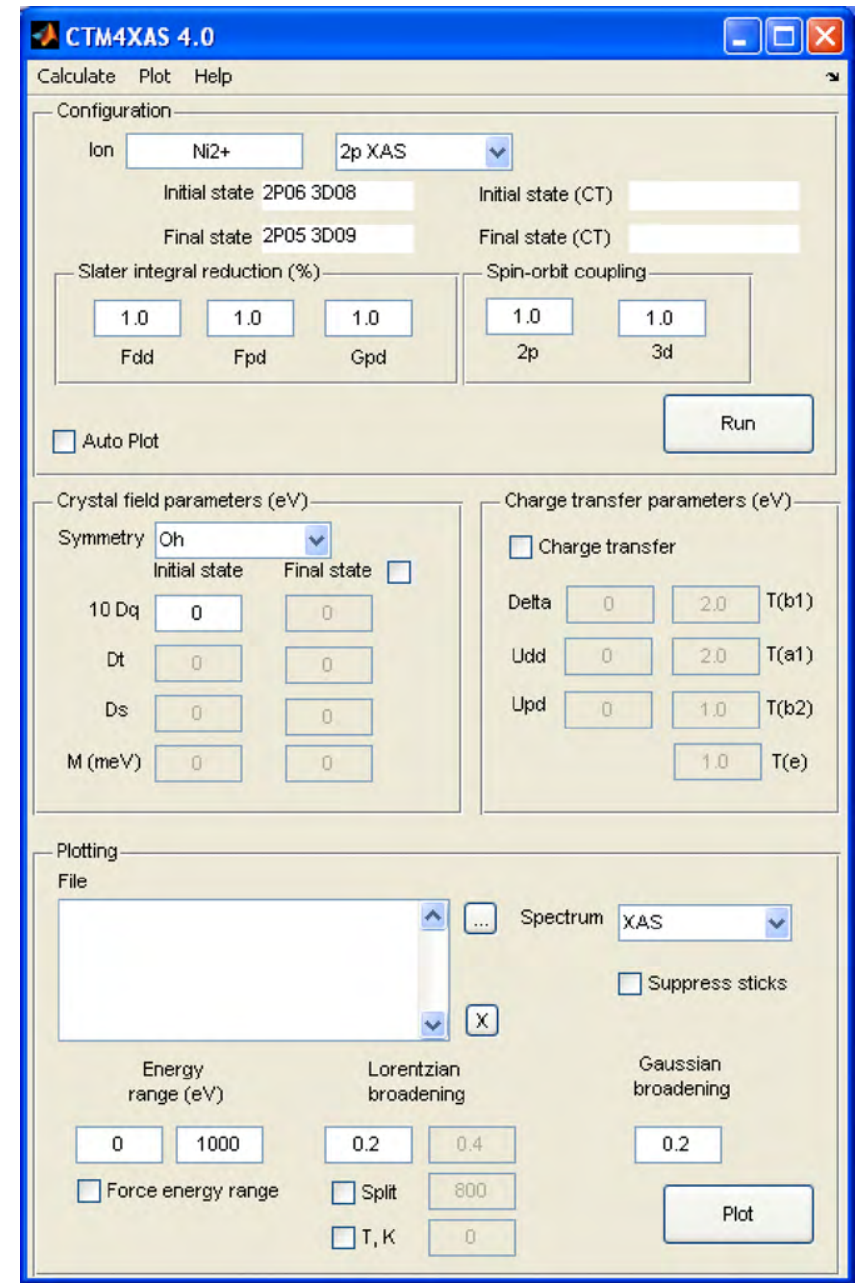

Fig. 1. The CTM4XAS program is based on a parameter based panel. The panel is divided in four sections, respectively, the configuration and the atomic calculations at the top, the crystal field parameters (middle, left), the charge transfer parameters (middle, right) and the plotting options (bottom). Enlarged sections of the panel are shown below.

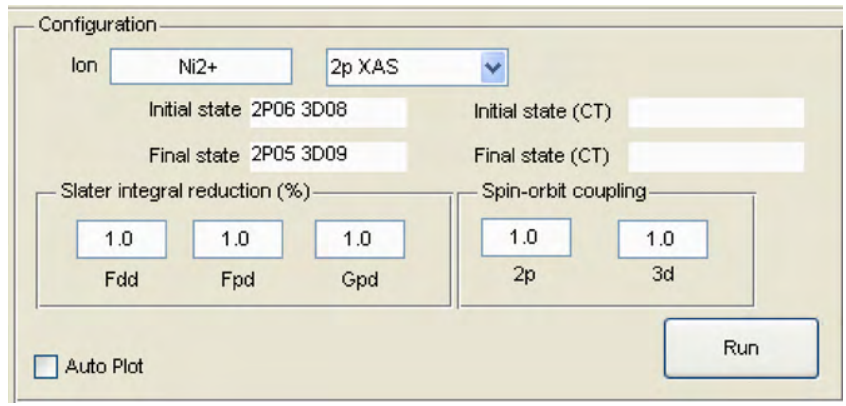

Fig. 2. The atomic multiplet calculation input of the CTM4XAS program.

and $3 p$ XAS, where $2 \mathrm{p}$ XAS is considered to be identical to $2 \mathrm{p}$ EELS. In case of $1 \mathrm{~s} X A S$, a quadrupole calculation is performed to simulate the pre-edge structure of $K$ edges. The $K$ edge itself should be calculated with DFT based computer codes. The XPS options include the $1 \mathrm{~s}, 2 \mathrm{~s}, 3 \mathrm{~s}, 2 \mathrm{p}$ and $3 \mathrm{p}$ spectra. XPS is automatically calculated with charge transfer because the XPS spectra are dominated by this effect, as will be discussed below. The two lines below give the initial state and final state configuration(s). If no charge transfer is included only one initial and final state is given. Further options include the $1 \mathrm{~s} 2 \mathrm{p}$ and $1 \mathrm{~s} 3 \mathrm{p}$ XES spectra.

The bottom part of the atomic multiplet section allow the control of the atomic parameters, respectively, the two-electron integrals coupling the $3 \mathrm{~d}$ electrons with each other and coupling the core state with the valence states and the spin-orbit coupling of the $2 \mathrm{p}$ core state and the $3 \mathrm{~d}$ valence state. The two-electron integrals are described with Slater integrals (Cowan, 1981). The $F_{\text {dd }}$ Slater integrals $\left(F^{2}\right.$ and $\left.F^{4}\right)$ are controlled by the first reduction factor. A parameter value of 1.0 implies the use of the atomic values, while a parameter value of 0.0 implies that the $F_{\mathrm{dd}}$ Slater integrals are set to zero. The atomic parameters are calculated with the Cowan program within the Hartree-Fock limit. A standard reduction to $80 \%$ of the Hartree-Fock values is assumed to represent the atomic values, as confirmed by experiment. Intermediate values imply the reduction of the $F_{\mathrm{dd}}$ Slater integral with the number given. A number can de chosen between 0.0 and 1.5 . The second parameter controls the $F_{\mathrm{pd}}$ Slater integral $\left(F^{2}\right)$ that couples the core and valence electrons. The third parameter controls the $G_{\text {pd }}$ exchange Slater integrals $\left(G^{1}\right.$ and $\left.G^{3}\right)$. In case of a $1 \mathrm{~s} / 2 \mathrm{~s} / 3 \mathrm{~s}$ core hole in the XPS calculations, only the $G^{1}$ value is different from zero.

The spin-orbit parameters work similarly. The core spin-orbit coupling is, in practice, never screened, so one should always use the default value of 1.0 for spectral simulations. It can be controlled only for didactical purposes. By setting the $2 \mathrm{p}$ spin-orbit coupling to zero, the $L_{3}$ and $L_{2}$ edge overlap and the, virtual, overall L edge spectral shape is found. The control over the $3 \mathrm{~d}$ spin-orbit coupling is a crucial parameter. Many 3d transition metal systems have an L edge that is better described with the $3 \mathrm{~d}$ spin-orbit coupling set to zero, though this is certainly not a general rule. For example $\mathrm{CrO}_{2}$ and $\mathrm{Fe}_{2} \mathrm{SiO}_{4}$ (de Groot et al., 2005) are better described without 3d spin-orbit coupling, while $\mathrm{LaTiO}_{3}$ and $\mathrm{CoO}$ (Haverkort et al., 2005) are better described with the inclusion of $3 \mathrm{~d}$ spin-orbit coupling. $\mathrm{CoO}$ and $\mathrm{CoF}_{2}$ show temperature dependent effects due to the $3 \mathrm{~d}$ spin-orbit coupling. The temperature of the simulation can be chosen in the plotting. All possible initial states are calculated, including the spectral shape of all these states. The temperature is included as a Boltzmann distribution over the initial state energy values. Switching the 'autoplot' button on, implies that the plot is directly shown on screen. If it is not switched on, the calculation is performed but no result is plotted. Clicking the RUN button start the charge transfer multiplet calculation. 

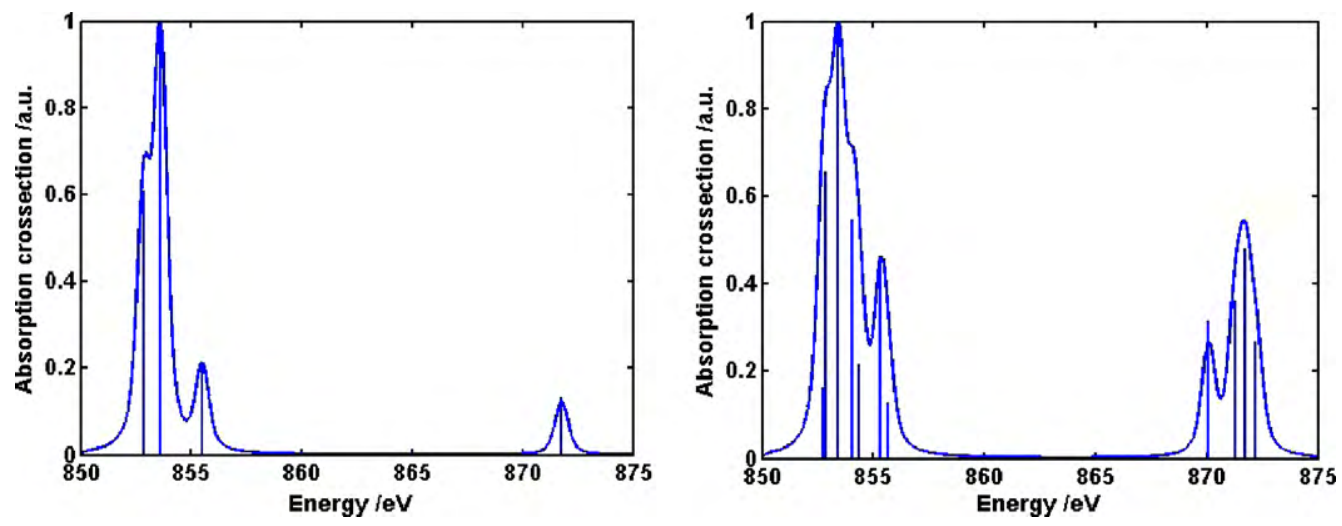

Fig. 3. (Left) Atomic multiplet calculation for $\mathrm{Ni}^{2+}$; (right) atomic multiplet calculation where the $3 \mathrm{~d}$ spin-orbit coupling has been set to zero.

\subsection{Example: atomic multiplet calculations of a $\mathrm{Ni}^{2+}$ ion}

As example, we provide the atomic multiplet calculation of a $\mathrm{Ni}^{2+}$ ion. $\mathrm{A} \mathrm{Ni}^{2+}$ ion has a $3 \mathrm{~d}^{8}$ ground state. The atomic couplings imply that the two holes will have the quantum numbers $m_{1}=-2$ and $m_{1}=-1$, both for a spin-down hole. This yields as Hunds rule ground state $L=3$ and $S=1$, in other words a ${ }^{4} \mathrm{~F}$ term symbol. Because the shell is more than half-full, after spin-orbit coupling the ground state has a $J$ value of 4 , i.e. ${ }^{3} \mathrm{~F}_{4}$ term symbol. The dipole selection rule states that the $J^{\prime}$ value in the final state must be 3 or 4 or 5 . The final state of $2 \mathrm{p}$ XAS has a $2 \mathrm{p}^{5} 3 \mathrm{~d}^{9}$ configuration, i.e. the coupling of a single $2 \mathrm{p}$ hole with a single $3 \mathrm{~d}$ hole. All term symbols of such configuration can be directly found by adding the $L$ and $S$ quantum numbers of the separate shells, i.e. $L_{\mathrm{TOT}}=3$ or 2 or 1 and $S_{\mathrm{TOT}}=1$ or 0 . All combinations are possible, yielding ${ }^{1} \mathrm{P},{ }^{1} \mathrm{D}$ and ${ }^{1} \mathrm{~F}$ states plus ${ }^{3} \mathrm{P},{ }^{3} \mathrm{D}$ and ${ }^{3} \mathrm{~F}$ states. This yields no possible term symbols with $J^{\prime}=5$, one term with $J^{\prime}=4\left({ }^{3} \mathrm{~F}_{4}\right)$ and three terms with $J^{\prime}=3\left({ }^{3} \mathrm{~F}_{3}\right.$, ${ }^{3} \mathrm{D}_{3}$ and $\left.{ }^{1} \mathrm{~F}_{3}\right)$. The result is an atomic multiplet spectrum with four peaks. Fig. 2 shows the result. Switching off the $3 \mathrm{~d}$ spin-orbit coupling implies that the ${ }^{3} \mathrm{~F}_{4}$ ground state becomes degenerate with the ${ }^{3} \mathrm{~F}_{3}$ and ${ }^{3} \mathrm{~F}_{2}$ states. The spectral shape is now given by all allowed transitions from $J=2$ or 3 or 4 . This implies that all final state $J$ from 1 to 5 are allowed.

It can be observed in Fig. 3 that the low $J^{\prime}$ values that were not allowed from the ${ }^{3} \mathrm{~F}_{4}$ state, can be found mainly at the $\mathrm{L}_{2}$ edge and at the high-energy side of the $\mathrm{L}_{3}$ edge. The binding energy of all edges in the CTM4XAS program has been set to the value as given in the X-ray Data Booklet (http://xdb.lbl.gov/). The binding energy of divalent ions has been set to the metal XPS binding energy. Trivalent ions have been shifted by $1.5 \mathrm{eV}$ to higher energy and subsequently each higher valence has been shifted by another $1.5 \mathrm{eV}$.

\subsection{Crystal field multiplet calculations}

The crystal field multiplet calculations are controlled in the middle left panel. The top button in Fig. 4 allows the choice of the point group symmetry. The symmetries are limited to octahedral $\left(\mathrm{O}_{\mathrm{h}}\right)$, tetragonal $\left(D_{4 h}\right)$ and fourfold symmetry $\left(C_{4}\right)$. De facto, this implies that also tetrahedral symmetry can be calculated for $2 \mathrm{p}$ and $3 \mathrm{p}$ XAS as this calculation is equivalent to an $\mathrm{O}_{h}$ calculation with negative crystal field values. In case of $1 \mathrm{~s}$ XAS pre-edges there is a large difference between octahedral and tetrahedral symmetry due to $4 \mathrm{p} 3 \mathrm{~d}$ mixing. This can be calculated with the multiplet program, but this option is not included in CTM4XAS. In $\mathrm{O}_{\mathrm{h}}$ symmetry, only the value of the cubic crystal field splitting 10Dq can be provided, as the other parameters are zero. In octahedral symmetry, $10 \mathrm{Dq}$ is defined as the energy difference between the $\mathrm{T}_{2 \mathrm{~g}}$ states and the $\mathrm{E}_{\mathrm{g}}$ states, neglecting all atomic parameters. The default settings assume the same

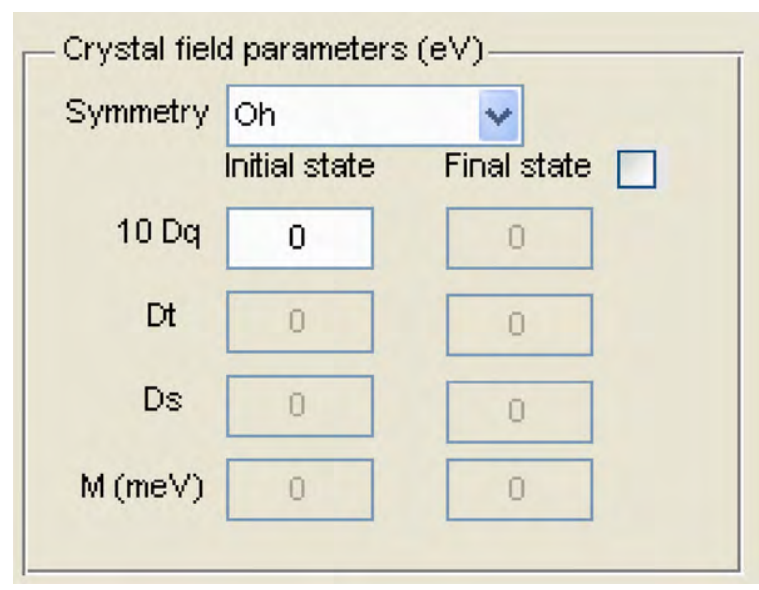

Fig. 4. The crystal field calculation input of the CTM4XAS program.

values for $10 \mathrm{Dq}$, Ds, Dt and $\mathrm{M}$ for the initial state and final state. One can choose different values if the final state tick mark is turned on.

The CTM4XAS program uses as input the optical parameters Dq, Ds and Dt (Lever and Solomon, 1999). The multiplet program is making use of a group theory program from Butler to perform the crystal field multiplet calculations (Butler, 1981). In the Butler program, another set of parameters are used, with in $\mathrm{D}_{4 \mathrm{~h}}$ symmetry, respectively, the $X_{40}, X_{42}$ and $X_{22}$ parameters, which are linear combinations of Dq. Ds and Dt. Ds $=-X_{22} / \sqrt{ } 70$ and $\mathrm{Dt}=-2 X_{42} / 5 \sqrt{ } 42$. The value of $\mathrm{Dq}$ is given as a combination of $X_{40}$ and $X_{42}: \mathrm{Dq}=X_{40} / 6 \sqrt{ } 30-7 X_{42} / 30 \sqrt{ } 42$. The output of the crystal field multiplet program is given in the ora file. The symmetry states are indicated in Butler's notation and Table 1 gives the relation to the more familiar Mulliken $\left(A_{1}, T_{1}\right.$, etc. $)$ and Bethe $\left(\Gamma_{1}, \Gamma_{2}\right.$, etc. $)$ notations. If the $\mathrm{C}_{4}$ symmetry option is selected an exchange field is added. The inclusion of the exchange field is used to describe the exchange field in ferromagnetic systems and as such it separates the magnetic substates. The direct consequence of the exchange field is the occurrence of a Magnetic Circular Dichroism (MCD)

Table 1

The labels for the configurations in octahedral symmetry ( $\mathrm{O}_{\mathrm{h}}$ group).

\begin{tabular}{lll}
\hline Butler & Mulliken & Bethe (Koster) \\
\hline 0 & $A_{1}$ & $\Gamma_{1}$ \\
$\hat{0}$ & $A_{2}$ & $\Gamma_{2}$ \\
2 & $E$ & $\Gamma_{3}$ \\
1 & $T_{1}$ & $\Gamma_{4}$ \\
$\hat{1}$ & $T_{2}$ & $\Gamma_{5}$ \\
\hline
\end{tabular}



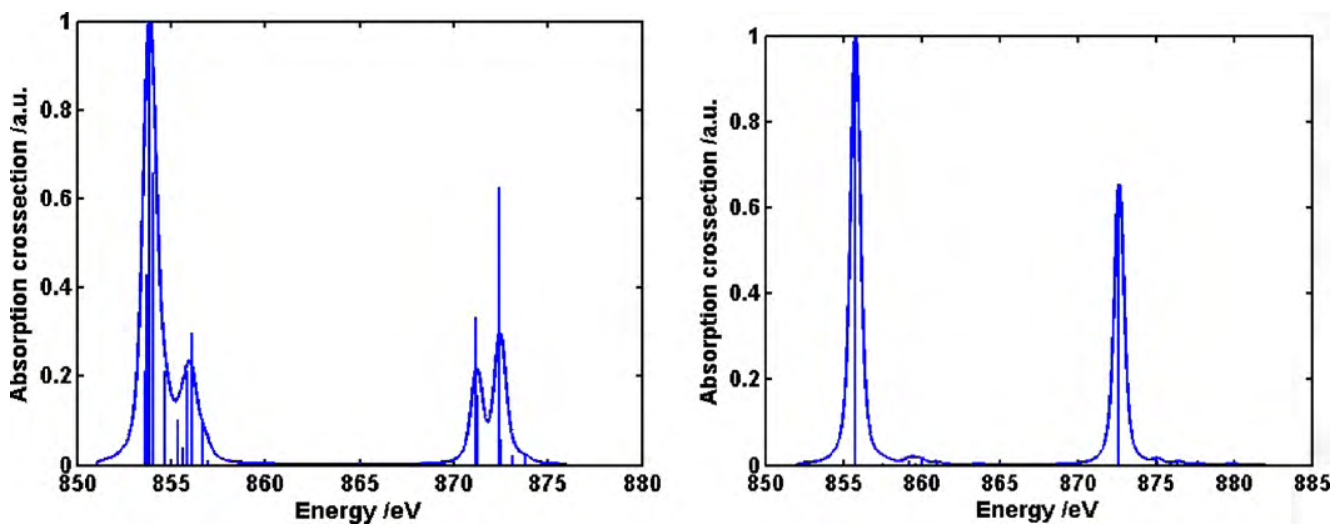

Fig. 5. Crystal field multiplet calculation for (left) $\mathrm{Ni}^{2+}$ with a $10 \mathrm{Dq}$ value of $1.0 \mathrm{eV}$; (right) $\mathrm{Ni}^{2+}$ with a $10 \mathrm{Dq}$ value of $5.0 \mathrm{eV}$ and values of $0.8 \mathrm{eV}$ for $\mathrm{Ds}$ and $\mathrm{Dt}$.

signal, defined as the difference in XAS by left, respectively, rightpolarized X-rays. Without the inclusion of an exchange field, the $\mathrm{X}-\mathrm{MCD}$ signal is always zero.

\subsection{The crystal field multiplet spectrum of a $\mathrm{Ni}^{2+}$ ion}

The atomic multiplet calculation of a $\mathrm{Ni}^{2+}$ ion can be extended by adding a non-zero $10 \mathrm{Dq}$ value. The inclusion of a cubic crystal field splits the ${ }^{3} \mathrm{~F}$ ground state into its ${ }^{3} \mathrm{~A}_{2},{ }^{3} \mathrm{~T}_{1}$ and ${ }^{3} \mathrm{~T}_{2}$ crystal field components. The crystal field effect splits a 3 d state into a $T_{2}$ and $E$ symmetry state. Acting on a $3 \mathrm{~d}^{8}$ configuration, the octahedral crystal field splits the ${ }^{3} \mathrm{~F}$ ground state into the crystal field components of an f-electron. An octahedral symmetry $3 \mathrm{~d}^{8}$ configuration has its $t_{2 g}$ shell filled and its $e_{g}$ shall half-filled, yielding a ${ }^{3} A_{2}$ ground state for all (positive) crystal field values. All crystal field calculations are performed in double group symmetry because of the large core hole spin-orbit coupling in the final state. $\mathrm{A}^{3} \mathrm{~A}_{2}$ ground state has $\Gamma_{5}$ double group symmetry, where we follow the rule that double group symmetries are indicated with Bethe notation; $\Gamma_{5}$ identifies with $T_{2}$ in Mulliken notation. Because this is a single state, the 3d spin-orbit coupling has no effect on the ground state. The $2 \mathrm{p}^{5} 3 \mathrm{~d}^{9}$ final state of the $2 \mathrm{p}$ XAS experiment has 25 possible states in Oh symmetry, 23 of which are allowed by the dipole selection rule. As such, one expects a crystal field multiplet spectrum containing 23 peaks, as shown in Fig. 5.

Using a $10 \mathrm{Dq}$ value of $1.0 \mathrm{eV}$ approximately yields the $\mathrm{NiO}$ spectrum, as can be seen in Fig. $5 . \mathrm{Ni}^{2+}$ in octahedral symmetry is always high-spin, but changing to $\mathrm{D}_{4 \mathrm{~h}}$ symmetry and using parameters representative of a square planar symmetry, one finds a low-spin ground state, as is found experimentally in $\mathrm{K}_{2} \mathrm{Ni}$-dithio-oxalate (van der Laan et al., 1988). The square planar $\left(D_{4 h}\right)$ symmetry shifts the $x^{2}-y^{2}$ orbital to high-energy, yielding a ground state with two holes in the $x^{2}-y^{2}$ orbital and all other orbitals double occupied. Adding an exchange field of $10 \mathrm{meV}$ combined with a cubic crystal field of $1.4 \mathrm{eV}$ reproduces the 2p EELS/XAS and its X-MCD spectrum of the molecular magnet $\mathrm{Cs}\left[\mathrm{NiCr}(\mathrm{CN})_{6}\right]$ (Arrio et al., 1996). Fig. 6 shows the 2p XAS spectrum and the difference between left and right circular polarized X-rays.

\subsection{Charge transfer multiplet calculations}

Charge transfer multiplet calculations are controlled in the middle right panel. With the charge transfer tick mark, one activates the charge transfer parameters. The physics behind these parameters is the charge transfer effect as initially developed for photoemission experiments (de Groot and Kotani, 2008). The left column gives the charge transfer energy $\Delta$, i.e. the energy between the $3 \mathrm{~d}^{8}$ and $3 \mathrm{~d}^{9} \mathrm{~L}$ configuration for a $\mathrm{Ni}^{2+}$ ion. The $U_{\mathrm{dd}}$ parameter defines the Hubbard $U$ value and the $U_{\text {pd }}$ parameter the core hole potential. In case of a two-configuration charge transfer multiplet calculation for XAS, $U_{\mathrm{dd}}$ has no direct influence and only the energy difference $U_{\mathrm{pd}}-U_{\mathrm{dd}}$ is important. In case of XPS calculations, $U_{\mathrm{dd}}$ has a direct influence on the spectrum. XPS calculations are always performed with the charge transfer effect switched on. The right column of parameters define the hopping terms that are defined for the four different symmetries in $\mathrm{D}_{4 \mathrm{~h}}$ symmetry, respectively $B_{1}, A_{1}, B_{2}$ and $E$ symmetry, related to, respectively, the $x^{2}-y^{2}, z^{2}, x y$ and $x z / y z$ orbitals. In $\mathrm{O}_{\mathrm{h}}$ symmetry the $B_{1}$ and $A_{1}$ parameters must be equal and relate to $E$ symmetry; the $B_{2}$ and $E$ parameters also are equal and relate to $T_{2}$ symmetry. In octahedral transition metal oxides the hopping
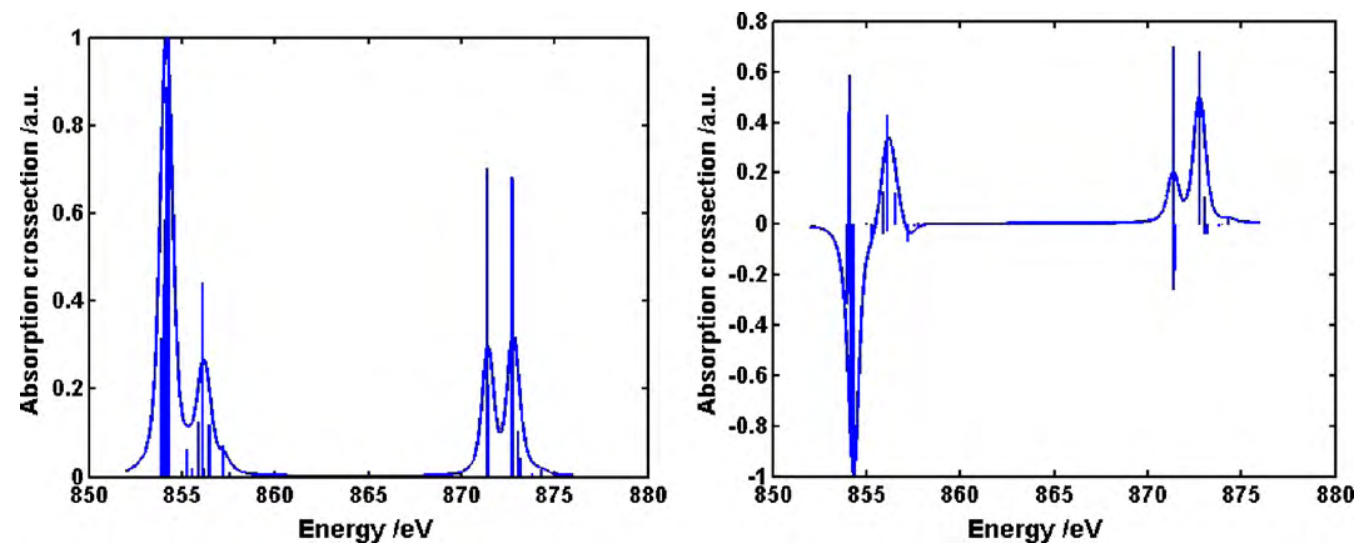

Fig. 6. Crystal field multiplet calculation for (left) $\mathrm{Ni}^{2+}$ with a $10 \mathrm{Dq}$ value of $1.4 \mathrm{eV}$ and an exchange field of $10 \mathrm{meV}$; (right) the corresponding $\mathrm{X}-\mathrm{MCD}$ spectrum. 


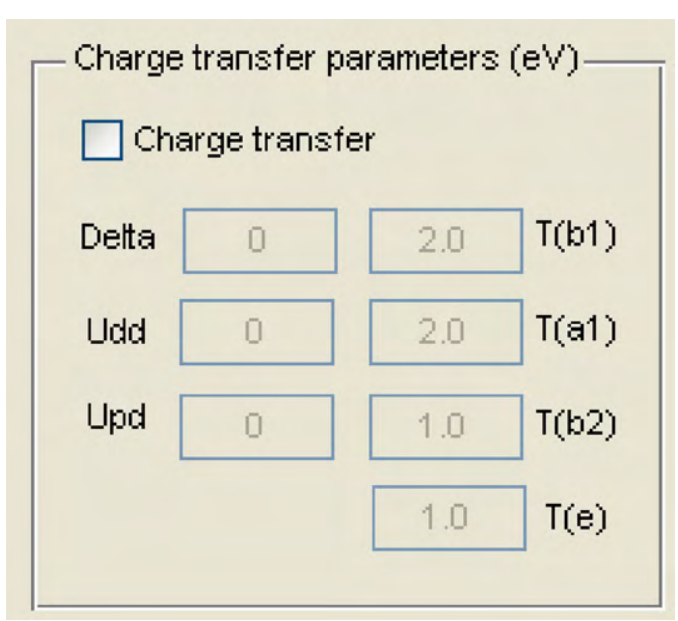

Fig. 7. The charge transfer calculation input of the CTM4XAS program.

parameter values are approximately given by the values $2.0,2.0$, 1.0 and 1.0. These values constitute the default values as provided by the program (Fig. 7).

\subsection{The charge transfer multiplet spectrum for $a \mathrm{Ni}^{2+}$ ion and a $\mathrm{Cu}^{3+}$ ion}

We here discuss the charge transfer multiplet spectrum for a $\mathrm{Ni}^{2+}$ and $\mathrm{a} \mathrm{Cu}^{3+}$ ion, both consisting of a ground state given as a linear combination of $3 \mathrm{~d}^{8}$ and $3 \mathrm{~d}^{9} \mathrm{~L}$. The NiO $2 \mathrm{p}$ EELS spectrum contains a few peaks at energies in between the $L_{3}$ and $L_{2}$ edge. The inclusion of the charge transfer excitation can reproduce these peaks and, in addition provide an improved fit of the main $\mathrm{L}_{3}$ edge. However, the 2p EELS spectra of divalent transition metal ions are already well described without charge transfer. Charge transfer effects are crucial for the calculation of the XPS spectra as will be discussed in Section 3. Fig. 8 (left) shows the charge transfer multiplet spectrum of $\mathrm{NiO}$ with the charge transfer energy $\Delta$ equal to $3 \mathrm{eV}, U_{\mathrm{dd}}=6$ and $U_{\mathrm{pd}}=8$. The default hopping parameters are used. One observes a spectrum similar to the crystal field multiplet calculation as was shown in Fig. 5, with as additional feature a small charge transfer satellite at $860 \mathrm{eV}$. Because the hopping is different for $\mathrm{T}_{2 \mathrm{~g}}$ and $\mathrm{E}_{\mathrm{g}}$ states, this contributes an additional crystal field splitting. To correct for this effect, the ionic crystal field parameter $10 \mathrm{Dq}$ is reduced by $0.3 \mathrm{eV}$.

Charge transfer effects are for some systems very clearly observed in their $2 p$ EELS spectra. This is in particular the case for transition metal ions with a valence of three or four. A divalent transition metal ion has only lost its $4 \mathrm{~s}$ electrons, but increasing

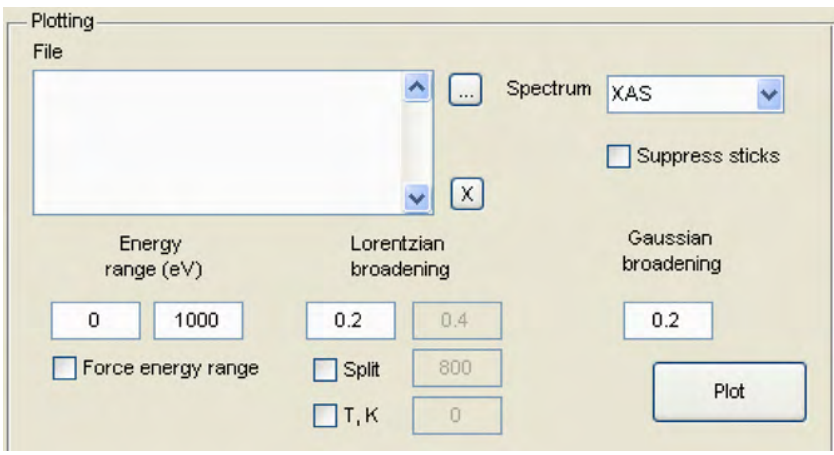

Fig. 9. The input of the plotting options for CTM4XAS.

the valence to trivalent implies that a $3 d$ electron is lost. This effect is compensated by short bond lengths hence by strong charge transfer effects. In case of $\mathrm{Cu}^{3+}$ the charge transfer is between $3 \mathrm{~d}^{8}$ and $3 \mathrm{~d}^{9} \mathrm{~L}$ configurations. In fact, for $\mathrm{Cu}^{3+}$ the charge transfer parameter is slightly negative implying that the $3 \mathrm{~d}^{9} \underline{\mathrm{L}}$ configuration has the lowest energy. The formal valence is still $3+$ because the spin state of the transition metal ion can best be described with $S=1$ or $S=0$ for, respectively, high-spin and low-spin $\mathrm{Cu}^{3+}$ systems.

Fig. 8 shows the simulation of a $\mathrm{Cu}^{3+}$ ion with $\Delta$ equal to $-1.3 \mathrm{eV}$, $U_{\mathrm{dd}}=6$ and $U_{\mathrm{pd}}=8$, using the default hopping terms $(\mathrm{T})$ and a $10 \mathrm{Dq}$ value of $1.0 \mathrm{eV}$. One can observe a single leading peak at the $\mathrm{L}_{3}$ edge that relates mainly to the $2 \mathrm{p}^{5} 3 \mathrm{~d}^{10} \mathrm{~L}$ final state. At higher energy a multiplet structure is observed that is mainly due to the $2 \mathrm{p}^{5} 3 \mathrm{~d}^{9}$ final states. The experimental spectrum of the high-spin $\mathrm{Cu}^{3+}$ systemCsKCuF 6 is very similar to this calculated spectrum ( $\mathrm{Hu}$ et al., 1998a,b).

\subsection{The plotting option in CTM4XAS}

Fig. 9 gives the bottom of the CTM4XAS interface. This part only takes care of the plotting options. The files that are calculated appear in the main plotting box. Files can be added to this list with the . . . button or removed with the $\mathbf{X}$ button. All files that are indicated in the box are plotted. This implies that if edges of different elements are indicated, the energy range contains both edges. The provided plots are only intended as an initial guide to the spectral shape. Each calculation has its output given as a X-file containing the spectral shape of the three polarization directions. The XAS/EELS spectrum can be plotted, or alternatively the MCD or MLD spectrum. Plotting the MCD spectrum implies the plot of the right-polarized X-rays minus the left-polarized X-rays. The MLD plots the left + right-2z-polarized spectrum. Alternatively one can manipulate the polarized spectra using the output file that con-
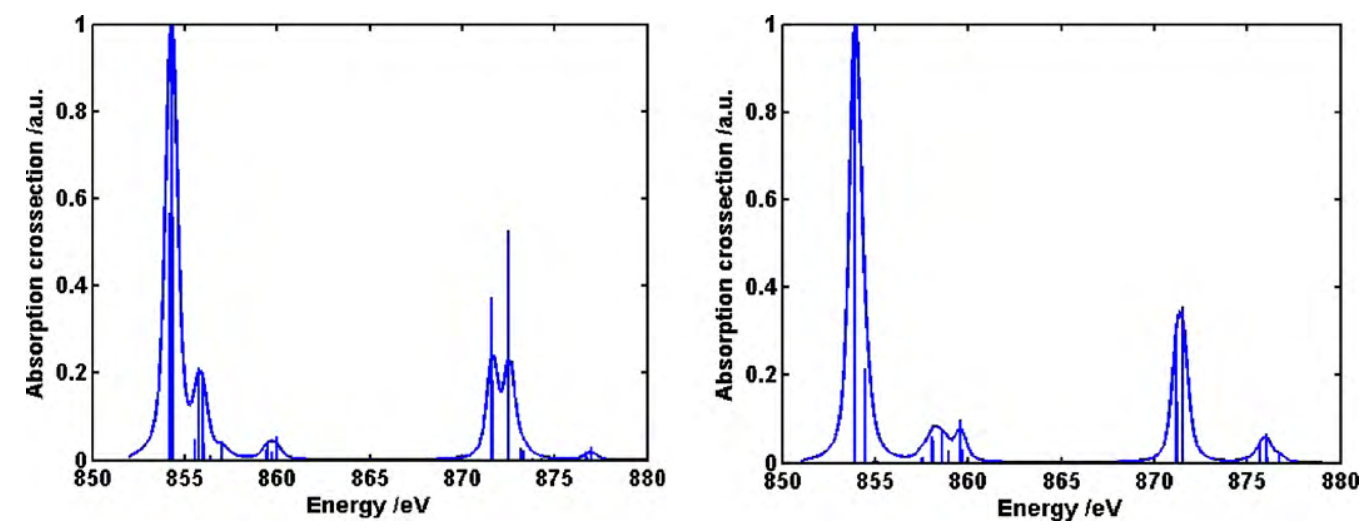

Fig. 8. Charge transfer multiplet calculation for (left) $\mathrm{Ni}^{2+}$ with the parameters set to NiO; (right) $\mathrm{Cu}^{3+}$ with $\Delta=-1.3 \mathrm{eV}$ and all parameters equal to the NiO case. 

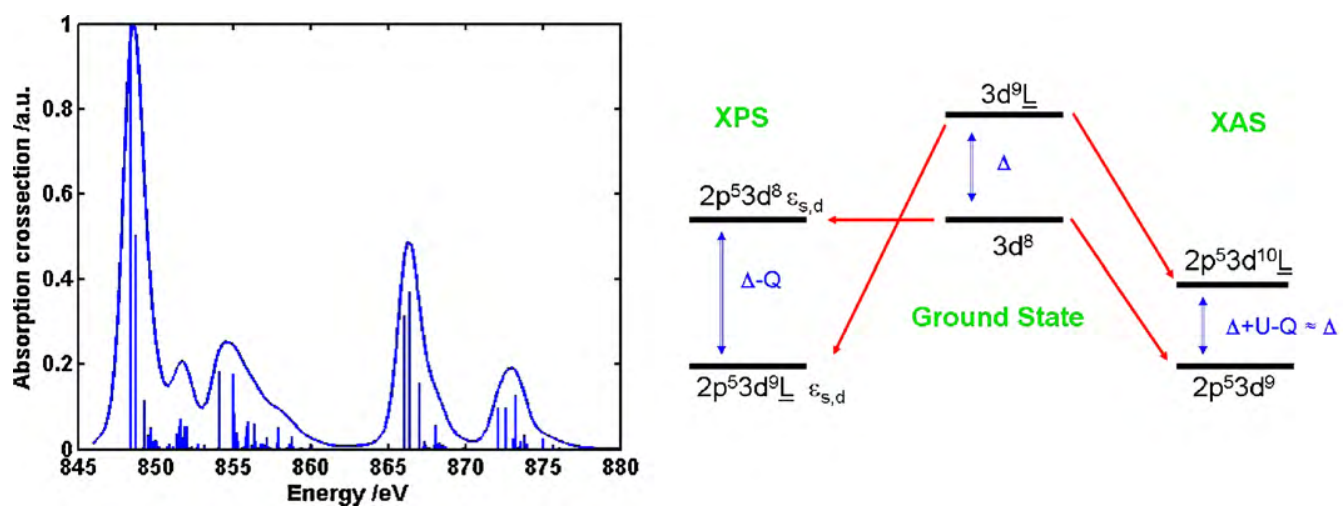

Fig. 10. Charge transfer multiplet for (left) the $2 \mathrm{p}$ XPS spectrum of $\mathrm{Ni}^{2+}$ with a $10 \mathrm{Dq}$ value of $1.4 \mathrm{eV}$; (right) the ordering of states for $2 \mathrm{p}$ XPS, respectively, $2 \mathrm{p} \mathrm{XAS} / \mathrm{EELS}$.

tains the three polarizations independently. As default, a single Lorentzian broadening of $0.2 \mathrm{eV}$ half-width half-maximum is used. One can modify this number and also split the spectrum into two parts, for example the $\mathrm{L}_{3}$ and $\mathrm{L}_{2}$ part, and broaden each part independently, using the split button and giving the energy in $\mathrm{eV}$. The default value for the Gaussian broadening is also set to $0.2 \mathrm{eV}$ halfwidth half-maximum. The default energy range is from the lowest energy till the highest energy. In case of large core hole spin-orbit splitting, one would prefer to have a more limited energy range, which can be set with the 'Force energy range' button, where the lowest and highest energy should be provided. The temperature can be added in Kelvin, if appropriate for the experiment. The sticks can be suppressed in the plot with the 'Suppress sticks' tick mark. These plotting options yield the most common spectra. If more complex spectra are required, one has to use the output files of the calculation and provide another program to plot the spectra. For example, plots as a function of temperature or for excited states can be plotted in this manner.
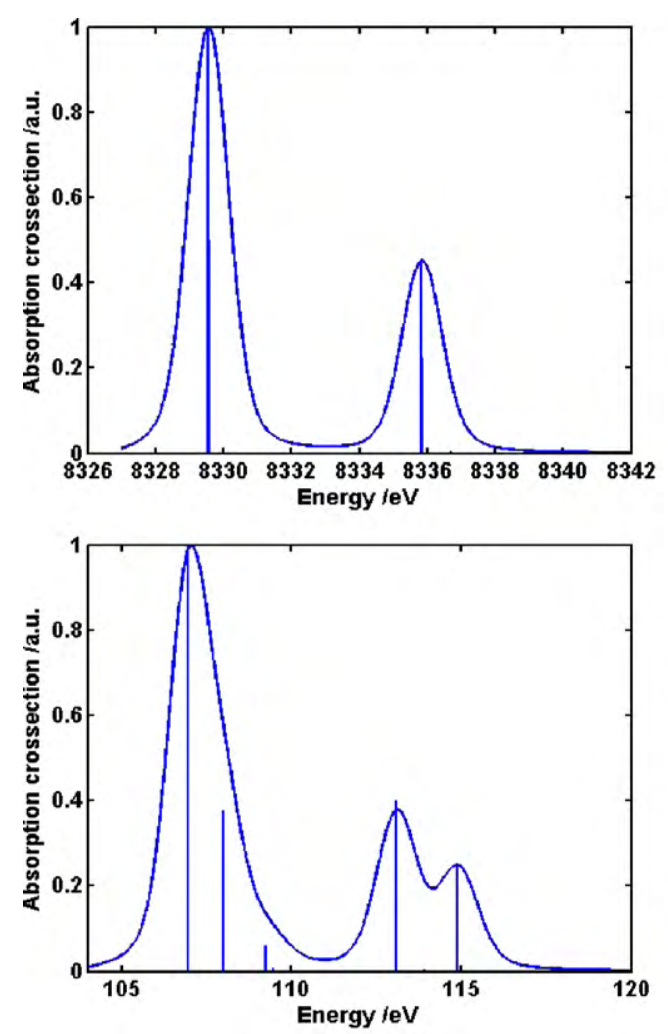

\section{Calculating XPS spectra with the CTM4XAS program}

The calculation of a $2 p$ XPS spectrum is analogous to $2 p$ XAS/EELS. One chooses the 2p XPS tab from the list and for the rest all parameters can remain the same. If one chooses XPS, the charge transfer tick mark is switched on automatically. We have chosen for charge transfer as a default option as it makes no physical sense to calculate an XPS spectrum without charge transfer. If one really would like to calculate an XPS spectrum without charge transfer, for example, for didactical purposes, one can set all hopping terms to zero, thereby decoupling the two configurations $3 \mathrm{~d}^{8}$ and $3 \mathrm{~d}^{9} \underline{\mathrm{L}}$. Then by setting a positive or negative charge transfer energy $\Delta$ one can create a pure $3 \mathrm{~d}^{8}$, respectively $3 \mathrm{~d}^{9} \underline{L}$ ground state and calculate its spectral shape.

Fig. 10 shows the $2 \mathrm{p}$ XPS spectrum of NiO, using exactly the same parameters as used for the 2p XAS/EELS spectrum in Fig. 5. The main difference between $2 p$ XAS/EELS and $2 p$ XPS is the large charge transfer effect in the latter. The final state of $2 p$ XPS contains
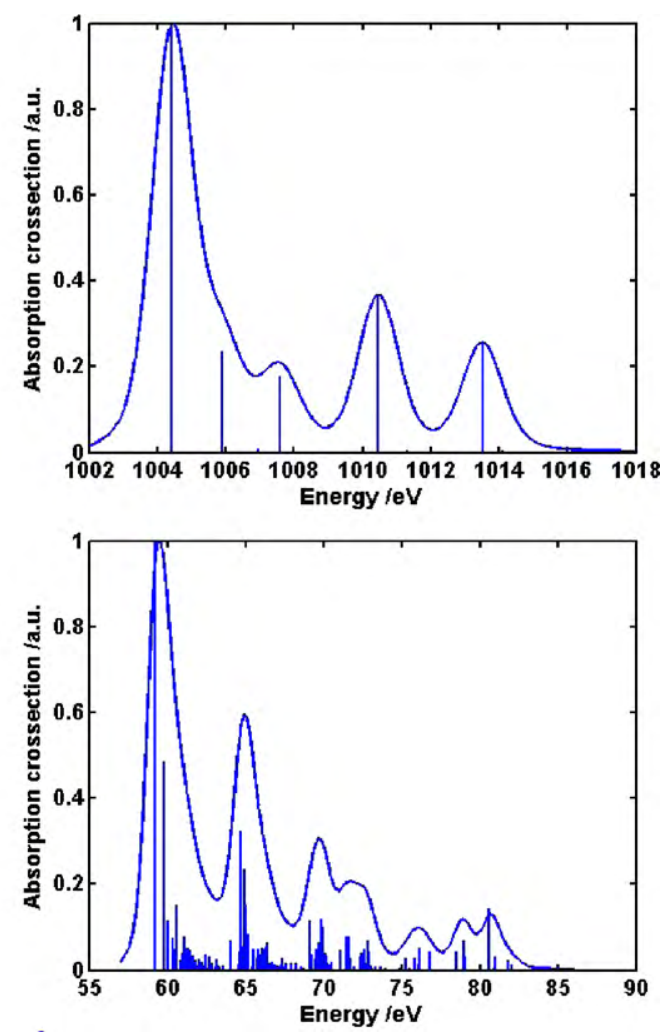

Fig. 11. Charge transfer multiplet calculations for $\mathrm{Ni}^{2+}$ using the NiO parameters; (top, left) 1s XPS; (top, right) 2s XPS; (bottom, left) 3s XPS and (bottom, right) 3p XPS. 
the configurations $2 \mathrm{p}^{5} 3 \mathrm{~d}^{8} \varepsilon_{\mathrm{s}, \mathrm{d}}+2 \mathrm{p}^{5} 3 \mathrm{~d}^{9} \underline{\mathrm{L}} \varepsilon_{\mathrm{s}, \mathrm{d}}$. The excited electron is assumed to be a free electron with s or $\mathrm{d}$ character $\left(\varepsilon_{\mathrm{s}, \mathrm{d}}\right)$. The energy of the $2 \mathrm{p}^{5} 3 \mathrm{~d}^{9} \mathrm{~L} \varepsilon_{\mathrm{s}, \mathrm{d}}$ configuration is shifted down due to the core hole potential $U_{c d}$, modifying the energy difference between the two configurations from $\Delta$ in the initial state to $\Delta-U_{\mathrm{cd}}$ in the final state. In case of $\mathrm{NiO}$, the energy changes from $+3.0 \mathrm{eV}$ to $-5.0 \mathrm{eV}$. This reordering of states implies that the final states at the lowest energy are dominated by $2 \mathrm{p}^{5} 3 \mathrm{~d}^{9} \mathrm{~L} \varepsilon_{\mathrm{s}, \mathrm{d}}$ character. Fig. 10 (right) gives the energy positions of the $3 \mathrm{~d}^{8}+3 \mathrm{~d}^{9} \underline{\mathrm{L}}$ initial state and its transition in $2 \mathrm{p}$ XPS, respectively, $2 \mathrm{p}$ XAS/EELS.

A more detailed analysis of the 2p XPS spectrum is possible with the inclusion of more than two configurations. This option is not included in the CTM4XAS interface, because the calculations would require considerable computer power. Charge transfer calculations with more than two configurations must be performed with dedicated multiplet programs. In case of XAS/EELS one can always neglect this third configuration (as far as the spectral shape is concerned) because its energy above the lowest energy state is high in both the initial and final state.

Once the charge transfer parameters are chosen one can calculate the complete set of XPS and XAS/EELS spectra. Provided that the two configuration calculation gives a good simulation of the XPS spectra, one can use the spectral shapes off all XAS/EELS and XPS spectra to optimize the charge transfer and crystal field parameters. The same set of ground state parameters should be able to simulate all XAS/EELS and XPS spectra. Fig. 11 gives the set of 3p, $1 \mathrm{~s}, 2 \mathrm{~s}$ and $3 \mathrm{~s}$ XPS for NiO, using the same parameters as used for $2 \mathrm{p} \mathrm{XAS/EELS} \mathrm{and} 2 \mathrm{p}$ XPS as given above. The $1 \mathrm{~s}$ XPS spectrum is only affected by charge transfer effects. The 2s XPS and 3s XPS are essentially determined by a combination of charge transfer and the $2 s 3 d$, respectively, 3s3d exchange interaction. The $3 p$ XPS spectrum is in addition affected by the $3 \mathrm{p} 3 \mathrm{~d}$ multiplet effects and the $3 p$ spin-orbit coupling. The CTM4XAS calculations use essentially the same approach as was used in the papers from Okada, Kotani and Thole in the early nineties (Okada and Kotani, 1992a,b; Okada et al., 1992).

\section{Concluding remarks}

We have discussed the CTM4XAS program, which is intended to be used as an initial tool to calculate the L edge spectra of transition metal systems as well as their XPS and XES spectra. If detailed theoretical simulation is needed, it is strongly suggested to perform additional multiplet calculations, for example using one of the ab initio multiplet calculations that are being developed.

We have limited the calculations to the transition metals. Only two configurations, $3 \mathrm{~d}^{n}$ and $3 \mathrm{~d}^{n+1} \underline{\mathrm{L}}$, can be included. This is usually sufficient for the XAS/EELS spectra of most systems. Molecular systems that have strong metal-ligand charge transfer need an additional configuration $3 \mathrm{~d}^{n-1} \underline{\mathrm{L}}$ (Hocking et al., 2006, 2007). These 3-configuration calculations cannot be treated by the interface, but they can be performed by the underlying multiplet programs.

Future developments will include the calculation of Auger spectra as well as the calculation of resonant spectra, including resonant photoemission, resonant Auger and resonant X-ray emission. For these experiments new interface programs will be developed. It is possible to register for the use of the CTM4XAS program on our website (website, 2010). Registration implies that all new programs and major developments will be provided via email.

\section{Acknowledgements}

We acknowledge financial support from the Netherlands National Science Foundation (NWO/vici and NWO/veni programs).

\section{References}

Chen, C.T., Sette, F., 1989. Rev. Sci. Instrum. 60, 1616.

Krivanek, O.L., Ursin, J.P., Bacon, N.J., Corbin, G.J., Dellby, N., Hrncirik, P., Murfitt, M.F. Own, C.S., Szilagyi, Z.S., 2009. Philos. Trans. A 367, 3683-3697.

Williams, K.C., 1966. Proc. Phys. Soc. 87, 983.

Fomichev, V.A., Zimkina, T.M., Gribovskii, S.A., Zhukova, I.I., 1967. Fizika Tverdogo Tela 9, 1490-1492.

Nakai, S., Nakamori, H., Tomita, A., Tsutsumi, K., Nakamura, H., Sugiura, C., 1974. Phys. Rev. B 9, 1870.

Shin, S., Suga, S., Kanzaki, H., Shibuya, S., Yamaguchi, T., 1981. Solid State Commun. $38,1281$.

Gupta, R.P., Sen, S.K., 1974. Phys. Rev. B 10, 71.

Gupta, R.P., Sen, S.K., 1975. Phys. Rev. B 12, 15

Suga, S., Shin, S., Taniguchi, M., Inoue, K., Seki, M., Nakada, I., Shibuya, S., Yamaguchi, T., 1982. Phys. Rev. B 25, 5487.

Shin, S., Suga, S., Taniguchi, M., Kanzaki, H., Shibuya, S., Yamaguchi, T., 1982. J. Phys Soc. Jpn. 51, 906.

Kotani, A., Toyozawa, Y., 1974. J. Phys. Soc. Jpn. 37, 912.

Asada, S., Sugano, S., 1976. J. Phys. Soc. Jpn. 41, 1291.

Zaanen, J., Sawatzky, G.A., Fink, J., Speier, W., Fuggle, J.C., 1985. Phys. Rev. B: Condens. Matter 32, 4905-4913.

Butler, P.H., 1981. Point Group Symmetry, Applications, Methods and Tables. Plenum, New York.

Thole, B.T., Vanderlaan, G., Butler, P.H., 1988. Chem. Phys. Lett. 149, 295-299.

de Groot, F.M.F., Fuggle, J.C., Thole, B.T., Sawatzky, G.A., 1990a. Phys. Rev. B 41 928-937.

de Groot, F.M.F., Fuggle, J.C., Thole, B.T., Sawatzky, G.A., 1990b. Phys. Rev. B 42, 5459-5468.

van der Laan, G., 1991. J. Phys. Condens. Matter 3, 7443-7454.

van der Laan, G., Kirkman, I.W., 1992. J. Phys. Condens. Matter 4, 4189-4204.

Ogasawara, H., Kotani, A., Okada, K., Thole, B.T., 1991a. Phys. Rev. B 43, 854-859.

Ogasawara, H., Kotani, A., Potze, R., Sawatzky, G.A., Thole, B.T., 1991b. Phys. Rev. B 44, 5465-5469.

Hu, Z., Kaindl, G., Warda, S.A., Reinen, D., de Groot, F.M.F., Muller, B.G., 1998a. Chem. Phys. 232, 63-74.

Hu, Z., Mazumdar, C., Kaindl, G., de Groot, F.M.F., Warda, S.A., Reinen, D., 1998b. Chem. Phys. Lett. 297, 321-328.

Hocking, R.K., Wasinger, E.C., de Groot, F.M.F., Hodgson, K.O., Hedman, B., Solomon, E.I., 2006. J. Am. Chem. Soc. 128, 10442-10451.

Hocking, R.K., Wasinger, E.C., Yan, Y.L., deGroot, F.M.F., Walker, F.A., Hodgson, K.O. Hedman, B., Solomon, E.I., 2007. J. Am. Chem. Soc. 129, 113-125.

Cabaret, D., Mauri, F., Henderson, G.S., 2007. Phys. Rev. B, 75.

Gaudry, E., Cabaret, D., Sainctavit, P., Brouder, C., Mauri, F., Goulon, J., Rogalev, A., 2005. J. Phys. Condens. Matter 17, 5467-5480.

Cabaret, D., Bordage, A., Juhin, A., Arfaoui, M., Gaudry, E., 2010. Phys. Chem. Chem. Phys. 12, 5619-5633.

Juhin, A., de Groot, F., Vanko, G., Calandra, M., Brouder, C., 2010. Phys. Rev. B, 81

Gao, S.P., Pickard, C.J., Payne, M.C., Zhu, J., Yuan, J., 2008. Phys. Rev. B, 77.

Schwarz, K., Blaha, P., Madsen, G.K.H., 2002. Comp. Phys. Commun. 147, 71-76.

Joly, Y., 2003. J. Synchrotron. Radiat. 10, 58-63.

Rehr, J.J., Albers, R.C., 2000. Rev. Mod. Phys. 72, 621-654.

Kolczewski, C., Hermann, K., 2005. Theor. Chem. Acc. 114, 60-67.

George, S.D., Petrenko, T., Neese, F., 2008. J. Phys. Chem. A 112, 12936-12943.

Kruger, P., Natoli, C.R., 2004. Phys. Rev. B, 70.

Agui, A., Uozumi, T., Mizumaki, M., Kaambre, T., 2009. Phys. Rev. B 79, 4.

Ikeno, H., de Groot, F.M.F., Stavitski, E., Tanaka, I., 2009. J. Phys. Condens. Matter 21, 104208.

Haverkort, M., 2009. (priv. comm.).

de Groot, F., 2001. Chem. Rev. 101, 1779-1808.

de Groot, F., 2005. Coord. Chem. Rev. 249, 31-63.

de Groot, F., Kotani, A., 2008. Core Level Spectroscopy of Solids. Taylor and Francis, New York.

de Groot, F.M.F., 1994. J. Electron. Spectrosc. 67, 529-622.

CTM4XAS website, 2010. Send an email to f.m.f.degroot@uu.nl in order to obtain access to the CTM4XAS website.

Cowan, R.D., 1981. The Theory of Atomic Structure and Spectra. U. California Press, Berkeley.

de Groot, F.M.F., Glatzel, P., Bergmann, U., van Aken, P.A., Barrea, R.A., Klemme, S Hävecker, M., Knop-Gericke, A., Heijboer, W.M., Weckhuysen, B.M., 2005. J. Phys Chem. B 109, 20751-20762.

Haverkort, M.W., Hu, Z., Tanaka, A., Reichelt, W., Streltsov, S.V., Korotin, M.A., Anisimov, V.I., Hsieh, H.H., Lin, H.J., Chen, C.T., Khomskii, D.I., Tjeng, L.H., 2005. Phys. Rev. Lett., 95.

Lever, A.B., Solomon, E.I., 1999. Inorganic Electronic Structure and Spectroscopy, Chapter 1, Ligand Field Theory and The Properties of Transition Metal Complexes. Wiley, New York.

van der Laan, G., Thole, B.T., Sawatzky, G.A., Verdaguer, M., 1988. Phys. Rev. B 37 6587-6589.

Arrio, M.A., Sainctavit, P., Moulin, C.C.D., Brouder, C., de Groot, F.M.F., Mallah, T., Verdaguer, M., 1996. J. Phys. Chem. 100, 4679-4684.

Okada, K., Kotani, A., 1992a. J. Phys. Soc. Jpn. 61, 4619-4637.

Okada, K., Kotani, A., 1992b. J. Phys. Soc. Jpn. 61, 449-453.

Okada, K., Kotani, A., Thole, B.T., 1992. J. Electron. Spectrosc. 58, 325-343. 